\title{
Based Data Analysis of Automobile Reliability
}

\author{
XU Zhao \& TANG Meng-rou \\ Jiangxi Communications Vocational and Technical College, Nanchang, China
}

\begin{abstract}
In this paper, the point estimate method (including the general expectation estimation method and estimation of population variance method) interval and parameter estimation method for the reliability of auto parts for data analysis. Results show that the total expected estimation method is simple, but its accuracy is related to sample size; the overall variance estimation method is better than the overall expectation estimation method accuracy of estimation can be obtained, and the overall variance unbiased value; and the use of parametric interval estimation method is relatively accurate, but the calculation process is relatively complex.
\end{abstract}

KEYWORD: Automobile; reliability; data analysis

\section{SUMMARY}

Reliability is an emerging discipline. With the development of science and technology, the theory and application of reliability has been paid much attention in various technical fields who.

In the reliability analysis of automotive parts, generally not possible for all test for research object, but generally in the sample test, to study and estimate the overall condition of certain parts by the sample observations[1]. The experimental data, as shown in Figure 1 block diagram of the program on the components reliability analysis estimation.

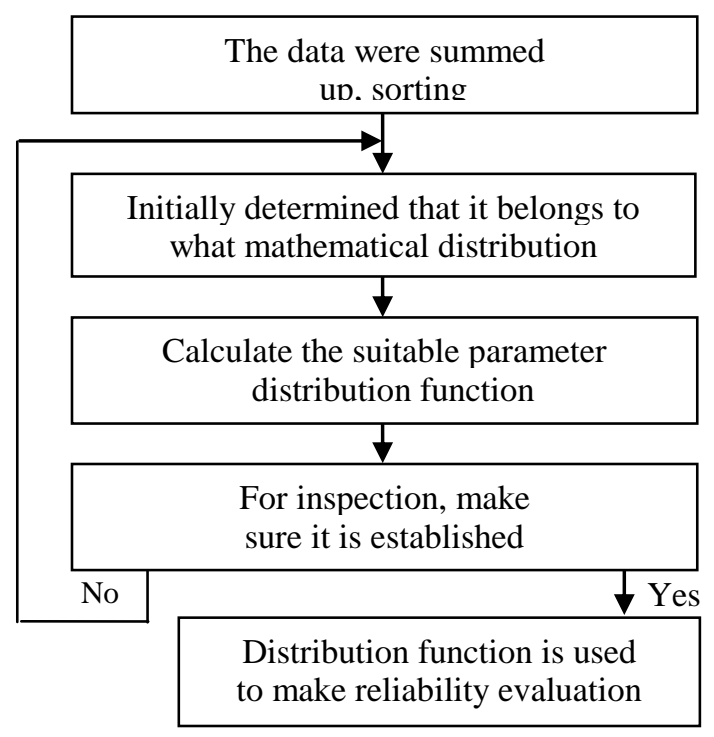

Figure 1. Reliability data analysis program

\section{POINT ESTIMATION}

Point estimation is used to observe a set of sample values, methods to estimate the unknown parameters of a general.

\subsection{Type area}

Samples from the overall, it must reflect the overall situation in a certain extent, so it can be used in the sample mean as the population mean, the $\mathrm{E}(\mathrm{X})$ of the estimated value, denoted as

$$
\bar{X}=E(X)
$$

Proof of theory and practice, to estimate the population mean is feasible using the sample mean, but the method of estimation accuracy and capacity of $n$ related. The sample size of $n$ is larger, higher precision of point estimation, estimated to be close to the population parameter; while the sample size $n$ is small, low value estimation precision, and is likely to have a greater difference with the actual[2]. For example, from a batch of qualified rate of car electric horn 99\%, selected 10 pieces of sample testing, if found to have 1 pieces of unqualified, qualified rate of point estimates of $\mathrm{P}=0.9$; if not found to be unqualified electric horn, $\mathrm{P}=1$. If the sample size is reduced, the representative of observations is worse, this is clearly inconsistent with the facts. 


\subsection{Of the overall variance estimation}

In general, the overall variance $\mathrm{D}(\mathrm{X})$ is unknown, can only use the sample variance $\sigma^{2}$ as an estimate of the population variance value. However, this estimate is not unbiased. Estimated to obtain the overall variance unbiased $S^{2}$ value, variance $\sigma$ samples must be $\sigma^{2}$ times the last related to the sample capacity of $\mathrm{n}$ modification coefficie $\beta$

$$
\beta=\frac{n}{n-1}
$$

So obtained without a poor estimate recipe

$$
S^{2}=\frac{n}{n-1} \sigma^{2}
$$

Unbiased standard deviation

$$
\mathrm{S}=\sqrt{\frac{n}{n-1}} \sigma \quad S=\sqrt{\frac{n}{n-1} \sum_{i=1}^{n}\left(X_{i}-\bar{X}\right)^{2}}
$$

The automobile headlamp as an example, the failure to obey normal distribution, i.e. $X \sim N\left(\mu, \sigma^{2}\right)$, the mean and variance of the overall distribution parameters are unknown. Randomly select a car 6 headlamp testing, to measure the life of the bulb is respectively: 1899, 1563, 1967, 1668, 1771, 1830 (h). We solve the population mean $\mu$, the overall variance unbiased estimate of $S^{2}$.

$$
\begin{aligned}
\mu= & \bar{X}=\frac{1}{6}(1899+1563+1967+1668+1771+1830)=1783 \\
S^{2}= & \frac{1}{6-1}\left[(1899-1783)^{2}+(1563-1780)^{2}+(1967-1783)^{2}\right. \\
& +(1668-1783)^{2}+(1771-1783)^{2} \\
& \left.+(1830-1783)^{2}\right]=21995.8
\end{aligned}
$$

\section{ESTIMATION OF PARAMETERS}

In order to overcome the point estimate method to estimate capacity than the hour not abuse, in the reliability data analysis of automobile parts, inter valestimation of parameters can be taken. This method not only use the sample point estimation on the overall parameters of $\theta$, but also estimate a interval, And calculate the interval containing the confidence level parameter $\theta$ is estimated, this form is called the interval estimation[3]. In fact, the interval estimation is to understand the unknown population parameter between statistics and what difference? Or, the unknown population parameter with much confidence falls in the interval given scope?

A general $\mathrm{X}$ distribution with an unknown parameter $\theta$, if the sample is determined by two statistics $\theta_{\mathrm{l}}$ and $\theta_{\mathrm{u}}$, for a given risk degree, $0<\alpha<1$ confidence level, it satisfies the following formul

$$
P\left(\theta_{l} \leq \theta \leq \theta_{u}\right)=1-\alpha
$$

Type in:

$\alpha$ - Degree of risk;

(1- $\alpha$ ) - Confidence level;

$\left(\theta_{\mathrm{l}} \leq \theta \leq \theta_{\mathrm{u}}\right)$-Confidence interval;

$\theta_{1}$-Upper confidence limit;

$\theta_{\mathrm{u}}$-The lower confidence limit.

Is called random interval $\left(\theta_{\mathrm{l}}, \theta_{\mathrm{u}}\right)$ is $\theta$ of $100 \times(1$ $\alpha) \%$ confidence interval. $\theta_{\mathrm{l}}$ and $\theta_{\mathrm{u}}$ respectivelyis $100 \times(1-\alpha) \%$ confidence lower limit and upper, simultaneously has the confidence of the lower and upper bounds, called two-sided confidence limit, as shown in Figure 2. $\mathbf{P}\left(\theta_{\mathrm{l}} \leq \theta \leq \theta_{\mathrm{u}}\right)$ for $\left(\theta_{\mathrm{l}}, \theta_{\mathrm{u}}\right)$ confidence level, the percentage is also called degree of confidence.

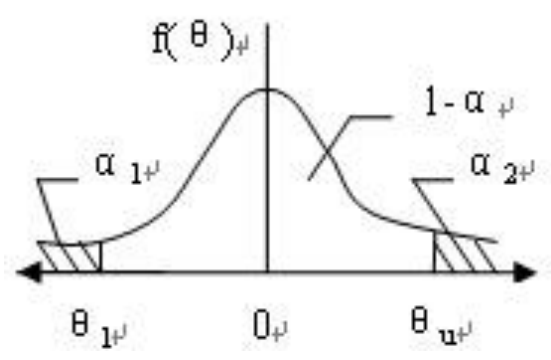

Figure 2. Two-sided confidence intervals

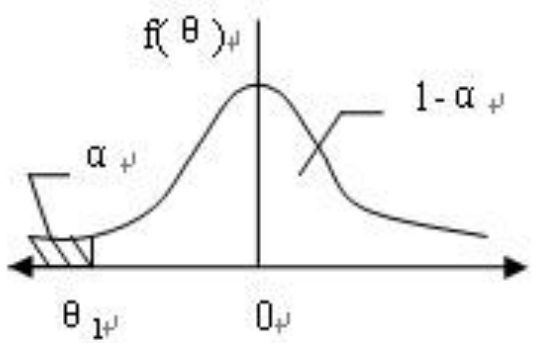

Figure 3. Only unilateral lower confidence limit

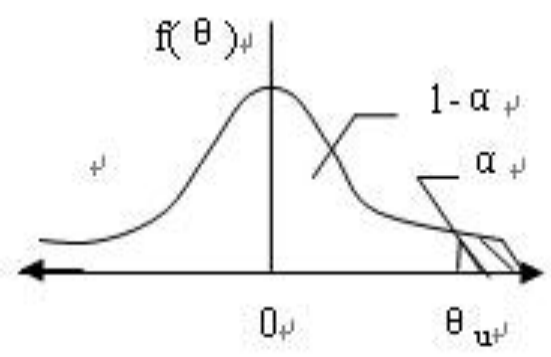

Figure 4. Confidence limit only unilateral upper limit

$\theta$ is unknown, its true value is constant, and $\theta_{1}$ and $\theta_{\mathrm{u}}$ is the random variable, the interval stochastic interval. For different sample observation value, we can get different interval, some interval contains $\theta$, some interval may not contain $\theta$. If the sample 100 times, including 98 times including $\theta$, the risk degree of $\alpha=0.02$, confidence level $(1-\alpha)=0.98$. Only the lower confidence limit or limit, called the one-sided confidence limit (in the study of reliability, the 
general attention lower confidence limit), as shown in Figure 3, shown in Figure 4. Said the confidence level for unilateral interv

$$
P\left(\theta_{l} \leq \theta\right)=1-\alpha
$$

\subsection{Interval estimation of a normal distribution of mean}

In practical work, the normal distribution has been widely used, particularly the application in the quality inspection and quality management is even more so. The normal distribution as the object, discusses the confidence interval of the mean and variance of $S^{2}$.

Let $\mathrm{X}_{1}, \mathrm{X}_{2}, \ldots \mathrm{X}_{\mathrm{n}}$ is subject to a sample of normal distribution, the sample mean

$$
\bar{X}=\frac{1}{n} \sum_{i=1}^{n} X_{i}
$$

Is an unbiased estimator of the population mean $\mu$, a point is estimated by $\mu$, the confidence interval for $\mu$

$$
\begin{aligned}
& \theta_{l}=\bar{X}-u_{\alpha / 2} \cdot \frac{S}{\sqrt{n}} \\
& \theta_{u}=\bar{X}+u_{\alpha / 2} \cdot \frac{S}{\sqrt{n}}
\end{aligned}
$$

For example, in the detection of automobile brake drumprocess, random sampled 6 , measured the diameter

were 279.96280.11280.05279.98280.02280.08 (mm), if the measured data obey normal distribution and the standard deviation is 0.03 , find the confidence of bilateral :

(1) $95 \%$ confidence interval;

(2)Normal distribution variance $\mathrm{S}$ is unknown, the confidence interval for the mean.

Solution: X said the number of sample length

(1)Calculation of the sample mean

$$
\begin{aligned}
\bar{X}= & \frac{1}{n} \sum_{i=1}^{n} X_{i}=\frac{1}{6}(279.96+280.11+280.05 \\
& +279.98+280.02+280.08)=280.03(\mathrm{~mm})
\end{aligned}
$$

(2)Calculation of $u_{a / 2}$, check the normal distribution function table $\mathrm{u}_{\mathrm{a} / 2}=1.96$

$$
\Phi\left(u_{a / 2}\right)=\frac{a}{2}=\frac{0.05}{2}=0.025
$$

Type: $\mathrm{u}_{\mathrm{a} / 2}$ given by the a based on the normal distribution function is obtained, which is to calculate the confidence interval

$$
\theta_{l}=\bar{X}-u_{\alpha / 2} \cdot \frac{S}{\sqrt{n}}=280.03-1.96 \times \frac{0.03}{\sqrt{6}}=280.01
$$

$\theta_{u}=\bar{X}+u_{\alpha / 2} \cdot \frac{S}{\sqrt{n}}=280.03+1.96 \times \frac{0.03}{\sqrt{6}}=280.05$

According to the calculation, by calculating the mean of 280.03 samples with $95 \%$ confidence, falls in the range (280.03280.03).

\subsection{Interval estimation of variance of normal distribution}

The discussion above estimates of mean interval, in practice also need each other difference for interval estimation, which for the analysis and reliability study of precision parts, automobile quality management, very useful.

Let $X_{1}, X_{2}, \ldots X_{n}$ is a general $X \sim N\left(\mu, \sigma^{2}\right)$ of a sample, wherein the $\mu$ is known, to determine the confidence level (1-a) of the variance in D (X) confidence interv

In general, the overall variance $\sigma^{2}$ is unknown, the unbiased estimator needs to be modified by the coefficien $\left(\frac{n}{n-1}\right)$

$$
\begin{gathered}
S^{2}=\left(\frac{n}{n-1}\right) \frac{1}{n} \sum_{i=1}^{n}\left(x_{i}-\bar{x}\right)^{2} \\
S^{2}=\frac{1}{n-1} \sum_{i=1}^{n}\left(x_{i}-\bar{x}\right)^{2}
\end{gathered}
$$

Visible, the sample variance and the total variance the difference between the sampling number $\mathrm{n}$, when the sampling number $\mathrm{n}$ is $\infty$, the difference is reduced to zero. By the sample statistic (variance) and the overall variance is close to the level, is a degree of freedom (n-1) subject to $X^{2}$ distribution, the $\mathrm{X}^{2}$ distribution function table look up. The mathematical proof is as follows:

$$
\mathrm{X}^{2}=\frac{(n-1) S^{2}}{D(X)}
$$

The type of function: $X^{2}(n-1)$. The equality of 1-a for the given confidence level

$$
P\left\{X_{1-a / 2}^{2}(n-1) \leq \frac{(n-1) S^{2}}{D(X)} \leq X_{a / 2}^{2}(n-1)\right\}=1-a
$$

Solution of inequality within the brackets, can get the confidence interval variance

$$
\begin{aligned}
& \frac{(n-1) S^{2}}{X_{a / 2}^{2}(n-1)} \leq D(X) \leq \frac{(n-1) S^{2}}{X_{1-a / 2}^{2}(n-1)} \\
& \sqrt{\frac{(n-1)}{X_{a / 2}^{2}(n-1)} S} \leq \sqrt{D(X)} \leq \sqrt{\frac{(n-1)}{X_{1-a / 2}^{2}(n-1)} S}
\end{aligned}
$$

Lower confidence limit for the variances 
$\frac{(n-1) S^{2}}{X_{a / 2}^{2}(n-1)}=S_{l}^{2}$

Upper confidence limit for the variances

$$
\begin{aligned}
& \frac{(n-1) S^{2}}{X_{1-a / 2}^{2}(n-1)}=S_{u}^{2} \\
& S_{l}^{2} \leq D(X) \leq S_{u}^{2}
\end{aligned}
$$

Thus, the standard deviation confidence interval

\section{CONCLUSION}

Through the analysis, the reliability data analysis of automotive parts can be estimated by the method of point (including the general expectation estimation method and estimation of population variance method) interval and the estimation of parameters (including the interval of a normal distribution with mean estimation method and the variance of normal distribution interval estimation method). The general expectation estimation methodusing the sample mean to estimate the average value of simple overall, but the estimation precision is related to sample size, sample capacity is small, the estimation accuracy is low. Estimation of population variance method than the general expectation estimation precision is high, and the estimated total variance unbiased sample variance can be modified on the value of the. Interval by a normal distribution with mean interval estimate method to estimate and the variance of normal distribution interval estimation method can accurately to the automobile parts reliability data analysis, but the calculation process is a little more complex.

\section{REFERENCES}

[1] Sun Guilin. Reliability and safety. Beijing: Chemical Industry Press, 1996

[2] Wang Kai. Common machine parts reliability design. Beijing: Mechanical Industry Press, 1996

[3] Pu Vinda. Automobile reliability engineering. Beijing: Mechanical Industry Press, 1998 